\title{
Distinguishing the $4 q A$ and $4 q B$ variants is essential for the diagnosis of facioscapulohumeral muscular dystrophy in the Chinese population
}

\author{
Zhi-Qiang Wang ${ }^{1}$, Ning Wang ${ }^{1}$, Silvere van der Maarel $^{2}$, Shen-Xing Murong ${ }^{1}$ and Zhi-Ying Wu ${ }^{\star 1,3}$
}

Facioscapulohumeral muscular dystrophy (FSHD) is the third most common inherited muscular dystrophy with markedly clinical variability and complex genetic cause. Several reports pertaining to the Caucasian population have confirmed that there are $4 \mathrm{qA}$ and $4 q B$ variants of the $4 q$ ter subtelomere, and FSHD is uniquely associated with the $4 q A$ variant. However, few data relevant to the Chinese population have been published. In present paper, detailed clinical and genetic re-evaluations were performed in members of four special families who had been initially diagnosed as atypical or asymptomatic FSHD based only on the D4Z4 repeat length analysis. The FSHD-sized D4Z4 repeats in the probands from families 1,2 and 3 were identified as $4 q B$ variants. These patients were further confirmed as limb-girdle muscular dystrophy (LGMD2) or myotonic dystrophy (DM1) by molecular analyses. Specifically, we identified a 4qB variant on chromosome 10 in the healthy members of the fourth FSHD family with complex D4Z4 rearrangements of two exchanged repeat arrays. For the first time, we demonstrated in the Chinese population that D4Z4 contractions on the 4qB variant do not cause FSHD and 4qB variant on chromosome 10 might also represent intermediate structures in the transition from 4q to $10 q$. Furthermore, our results emphasize that D4Z4 repeat length analysis alone is not sufficient for the diagnosis of FSHD, especially when used as an exclusion criterion. This analysis should be accompanied by $4 \mathrm{qA} / 4 \mathrm{qB}$ variant determination and integrated chromosome assignments, especially in patients with obscure and unclassified myopathies similar to atypical forms of FSHD.

European Journal of Human Genetics (2011) 19, 64-69; doi:10.1038/ejhg.2010.143; published online 25 August 2010

Keywords: Facioscapulohumeral muscular dystrophy; genetic diagnosis; 4qA/4qB variants; chromosome assignment

\section{INTRODUCTION}

Facioscapulohumeral muscular dystrophy (FSHD, MIM 158900) is an autosomal dominant disorder that presents the third most common human muscular dystrophy, affecting skeletal muscles in a pattern that starts in the facial, shoulder and upper arm muscles and gradually progresses to the abdominal and foot-extensor muscles. ${ }^{1}$ Although FSHD can be diagnosed using well-established diagnostic criteria, ${ }^{2}$ there is extreme intrafamilial and interfamilial variability in age at onset, severity and progression of the disease. Moreover, other myopathies can present a clinical picture that resembles FSHD, resulting in misdiagnosis.

Because the genetic defect of FSHD has been mapped to the $4 \mathrm{q} 35$ subtelomere, it has been recommended that the clinical diagnosis be confirmed via the screening of contractions of D4Z4 repeat arrays located on a 4qter subtelomere. ${ }^{3-5}$ Furthermore, several reports have confirmed that there are $4 \mathrm{qA}$ and $4 \mathrm{qB}$ variants of the $4 \mathrm{q}$ subtelomere, and FSHD is uniquely associated with the $4 \mathrm{qA}$ variant. ${ }^{6-10}$ These findings add to the diagnostic challenge and pathogenetic complexity. ${ }^{11}$ Therefore, the correct diagnosis of FSHD depends on the ability to distinguish the $4 \mathrm{qA}$ and $4 \mathrm{qB}$ variants. However, all published data regarding the identification of the $4 \mathrm{qA}$ and $4 \mathrm{qB}$ variants are limited to the Caucasian population, and few are related to the Chinese population.
In a previous study, we detected the $4 \mathrm{qA}$ and $4 \mathrm{qB}$ variants in a group of patients that included those who had been formerly diagnosed with FSHD based exclusively on the D4Z4 repeats length analysis. ${ }^{12,13}$ Four families were identified as carriers of the $4 \mathrm{qB}$ variant, and one of them had a complex genetic constitution. To better characterize these patients and to confirm the correct diagnosis, we performed a detailed clinical and genetic re-evaluation. The results revealed that the individuals who carried the $4 \mathrm{qB}$ variant were previously misdiagnosed with FSHD. These results also confirmed that the $4 \mathrm{qB}$ variant does not cause FSHD in the Chinese population.

\section{MATERIALS AND METHODS}

\section{Subjects and PFGE-based DNA analysis}

PFGE-based analysis of D4Z4 repeats and the identification of $4 \mathrm{qA}$ and $4 \mathrm{qB}$ variants using probes p13E-11, 4qA and $4 \mathrm{qB}$ were performed as previously reported, in members of 55 clinically diagnosed Chinese FSHD families. ${ }^{6,12,13}$ Four families were found to have a FSHD-sized 4qB-type D4Z4 repeat. These families were subjected to further clinical examinations and molecular analyses. A ten-grade clinical severity (CS) scale score ${ }^{14}$ was used to evaluate the phenotypes of the affected individuals. To identify the chromosomal origin of these fragments, NotI-digested DNA was hybridized to the chromosome 4-specific probe B31, as previously reported. ${ }^{15}$ Informed consent was obtained from each patient (if $<18$ years of age, consent 
was obtained from their legal guardians). The research protocol was approved by the local Ethics Committee for medical research.

\section{Western blot analysis}

Western blot analysis of protein extracts from muscle biopsy specimens was performed using a calpain 3 rabbit polyclonal antibody (H-80; Santa Cruz Biotechnology Inc., Santa Cruz, CA, USA), a dysferlin rabbit monoclonal antibody (Epitomics Inc., Burlingame, CA, USA), a $\beta$-sarcoglycan rabbit polyclonal antibody (H-98; Santa Cruz Biotechnology Inc.) and a GAPDH mouse monoclonal antibody (Beyotime Inc., Jiangsu, China). The procedure was performed as previously described. ${ }^{16,17}$ The average density of the bands were visualized and analyzed by Quantity One 4.6.2 software (Bio-Rad Laboratories, Sundbyberg, Sweden).

\section{Analysis of the CTG repeat of DMPK}

Expansion of the CTG repeat in the $3^{\prime}$ untranslated region of DMPK was analyzed by standard polymerase chain reaction, denaturing polyacrylamide gel electrophoresis, silver staining and sequencing. The primers were designed based on the sequence of DMPK (Ensembl Gene ID: ENSG 00000104936): forward $5^{\prime}$-GCTCGAAGGGTCCTTGTAGC- ${ }^{\prime}$ and reverse $5^{\prime}$-CTTCCCAGGC CTGCAGTTTG- $3^{\prime}$. The copies of the CTG repeat were calculated from the length of the amplification product as follows: copies of the CTG repeat= (length of amplification product (bp)-flanking sequence $105 \mathrm{bp}$ )/3 bp.

\section{RESULTS}

Family 1

The proband was a 32-year-old female who developed lower limb weakness at age 10. Her family history was negative. Upon first examination at age 20 , she presented proximal muscle weakness in the lower limbs and slightly affected facial muscles. CK was $97 \mathrm{U} / 1$ (reference range, $<170 \mathrm{U} / \mathrm{l}$ ), and electromyography (EMG) was myopathic. A muscle biopsy showed mild dystrophic changes. Muscle fiber necrosis or ragged red fibers were not observed. The presence of one 36-kb 4q-type D4Z4 repeat gave rise to a diagnostic consideration of mild atypical FSHD (Figure 1a).

Thereafter, the progression of weakness was very slow, and she was still able to rise from a squat by herself. Manual muscle testing revealed mild limb-girdle muscle weakness and a slight deltoid muscle paresis without scapular winging. Tendon reflexes were absent, and sensory examination was normal. The CS score was 3.5. The 36-kb 4q-type $\mathrm{D} 4 \mathrm{Z} 4$ repeat was further identified as the $4 \mathrm{qB}$ variant (Figure $1 \mathrm{~b}$ ). Hybridization of probe B31 with NotI-digested DNA yielded a chromosome 4-derived fragment of $218 \mathrm{~kb} \quad(36 \mathrm{~kb}+182 \mathrm{~kb}$; Figure 1c), which confirmed that this $36-\mathrm{kb} 4 \mathrm{qB}$ variant was from chromosome 4. Therefore, we had to re-assess the patient's clinical manifestation and took into consideration limb-girdle muscular dystrophy (LGMD). Western blot analysis of the patient's biopsy specimen revealed abnormal expression of calpain 3 and dysferlin (Figure 1d), suggesting that she suffered from primary calpain 3 deficiency and secondary dysfelinopathy (LGMD2A).

\section{Family 2}

The proband was a 17-year-old female who developed body limb weakness at age 8 . She had progressive symmetric girdle myopathy spreading from her shoulders to her hips. Neurological examination revealed that her stance was hyperlordotic and her abdomen was slightly protuberant. Bilateral weakness of the extensors of the shoulders, knees and hips interfered with activities such as raising arms above the head, climbing stairs or rising from a stooped posture. The lip had some looseness and protrusion. Bilateral calf hypertrophies were also observed. CK was markedly elevated at $5460 \mathrm{U} / \mathrm{l}$, and EMG was myopathic. A muscle biopsy displayed moderately dystrophic features without obvious inflammatory infiltration. Her asymptomatic parents were consanguineous and her two elder sisters and one brother had died in their first decade of life. Her gender and myopathological features did not support Duchenne/Becker muscular dystrophy, and it was difficult to distinguish clinically whether the variable myopathic weakness was due to FSHD or LGMD. Thus, Southern blot analysis with probe p13E-11 was performed for this patient and the result revealed the presence of one 4q-type D4Z4 repeat of $31 \mathrm{~kb}$ (Figure 2a), initially confirming facial-sparing FSHD.

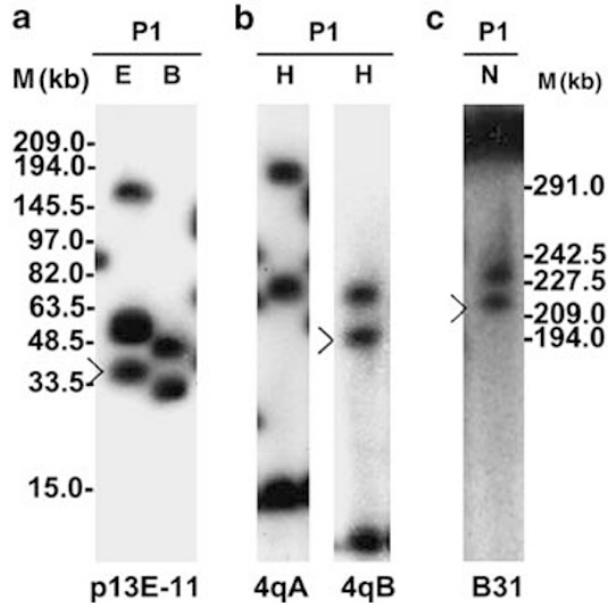

d
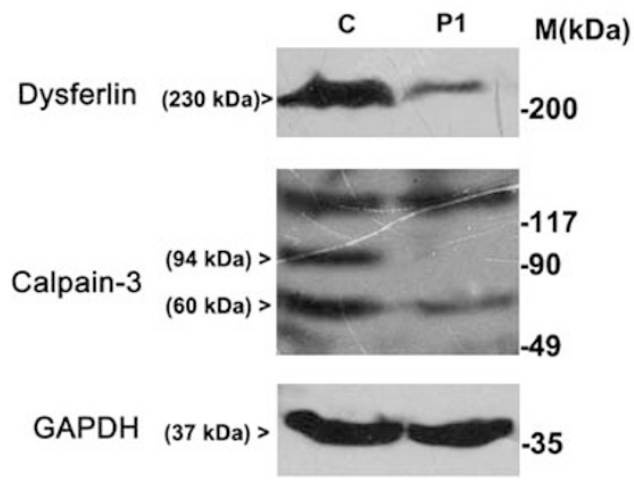

Figure 1 (a-c) PFGE-based Southern blot analysis of the proband (P1) from family 1. (a) DNA was digested with EcoRI (E) and EcoRI/BInI (B), subsequently hybridized with probe p13E-11. 4q-type alleles are resistant to $B|n|$, whereas $10 \mathrm{q}$-type alleles are sensitive to $B \mid n l$. The proband carried two $4 \mathrm{q}$-type alleles of $36 \mathrm{~kb}$ (arrow) and $50 \mathrm{~kb}$, and two 10q-type alleles of $52 \mathrm{~kb}$ and $157 \mathrm{~kb}$. (b) DNA was digested with HindIII (H) and hybridized with probes 4qA and 4qB. 4q-type alleles of $36 \mathrm{~kb}$ (arrow) and $50 \mathrm{~kb}$ were identified as 4qB variants. (c) DNA was digested with Notl (N) and hybridized with probe B31. The 4q-type alleles of $36 \mathrm{~kb}$ and $50 \mathrm{~kb}$ were visualized at $218 \mathrm{~kb}(36 \mathrm{~kb}+182 \mathrm{~kb}$; arrow) and $232 \mathrm{~kb}(50 \mathrm{~kb}+182 \mathrm{~kb})$, showing that the $36-\mathrm{kb} 4 \mathrm{qB}$ variant resided on chromosome 4. The size marker was the MidRange PFG marker (M). (d) Western blot analysis of muscle proteins. GAPDH was used as the internal control. Dysferlin is normally visualized as a 230-kDa band, and calpain 3 as $94-\mathrm{kDa}$ and $60-\mathrm{kDa}$ bands. Expression of dysferlin showed a significant decrease $(<30 \%$ of normal) in the muscle protein of the proband (P1) compared with that of the control (C). In the expression of calpain 3 , the $94-\mathrm{kDa}$ band was completely absent, and the $60-\mathrm{kDa}$ band was obviously reduced in the patient. 


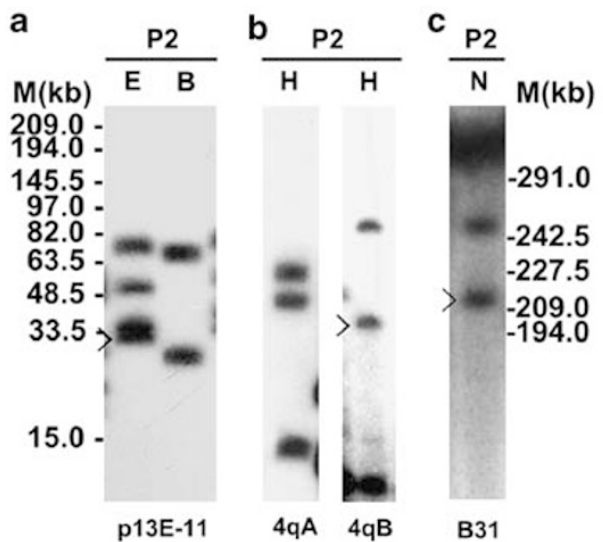

d

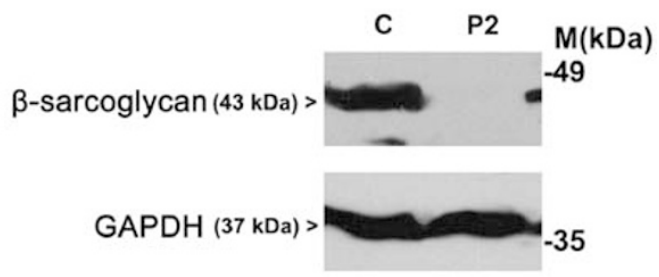

Figure 2 (a-c) PFGE-based Southern blot analysis of the proband (P2) from family 2. (a) The proband carried two 4q-type alleles of $31 \mathrm{~kb}$ (arrow) and $72 \mathrm{~kb}$, and two 10q-type alleles of $36 \mathrm{~kb}$ and $51 \mathrm{~kb}$. (b) Allelotyping identified one $4 \mathrm{qA}$ variant of $31 \mathrm{~kb}$ (arrow) and three $4 \mathrm{qB}$ variants of $72 \mathrm{~kb}$, $36 \mathrm{~kb}$ and $51 \mathrm{~kb}$. (c) Two $4 \mathrm{q}$-type alleles of $31 \mathrm{~kb}$ and $72 \mathrm{~kb}$ were both located on chromosome 4, as they were visualized at $213 \mathrm{~kb}(31 \mathrm{~kb}+182 \mathrm{~kb}$; arrow) and $254 \mathrm{~kb}$ $(72 \mathrm{~kb}+182 \mathrm{~kb})$. (d) Western blot analysis of muscle proteins. $\beta$-sarcoglycan is normally visualized as a 43-kDa band. Expression of $\beta$-sarcoglycan was absent in the proband (P2) compared with the control (C), suggesting that sarcoglycan deficiency caused LGMD2.

Recently, further haplotyping analysis showed that this 31-kb repeat was the $4 \mathrm{qB}$ variant (Figure $2 \mathrm{~b}$ ). After hybridization with probe $\mathrm{B} 31$, the chromosomal assignment revealed a NotI fragment of $213 \mathrm{~kb}$ $(31 \mathrm{~kb}+182 \mathrm{~kb})$, confirming that this $31-\mathrm{kb}$ fragment was on chromosome 4 (Figure 2c). We therefore reconsidered the diagnosis and analyzed the patient's dystrophin-associated glycoproteins. A $\beta$-sarcoglycan deficiency was confirmed by muscle protein analysis (Figure 2d), and she was finally diagnosed with severe childhood LGMD2E.

\section{Family 3}

The proband was a 38-year-old female who was referred for slowly progressing weakness of the arm and shoulder girdle in her second decade of life. On first neurological examination at age 35, ptosis of the eyelids, and thinness and slackness of the facial muscles were evident, along with mild weakness and atrophy of the shoulder girdle and pelvic girdle muscle without obvious scapular winging. Her parents both died in their sixties without signs of muscle problems. Her CK was slightly elevated at 370 U/l. EMG showed myopathic changes with a few myotonic discharges. A muscle biopsy performed in another hospital showed mild dystrophic changes. The initial molecular analysis showed one 36-kb 4q-type D4Z4 repeat compatible with the diagnosis of atypical FSHD.

Her symptoms continued to progress slowly, with gradual involvement of the proximal muscles of the lower limbs and the trunk. Specifically, she started to notice that strong clenching of the fist and closure of the eyelids were followed by a delay in relaxation. Moderate muscle weakness and myotonia of the hands and extensors of the forearms were also present on a neurological re-examination. Thus, we had to repeat the PFGE analysis with probes p13E-11, 4qA, 4qB and B31. The patient carried three BlnI-resistant D4Z4 repeats of $36 \mathrm{~kb}$, $76 \mathrm{~kb}$ and $86 \mathrm{~kb}$ (Figure $3 \mathrm{a}$ ), and the $36-\mathrm{kb}$ repeat was identified as the $4 \mathrm{qB}$ variant (Figure $3 \mathrm{~b}$ ). Finally, the $36-\mathrm{kb} 4 \mathrm{qB}$ variant was confirmed to reside on chromosome 4 , and the $86-\mathrm{kb}$ D4Z4 repeat was a mixture of $4 \mathrm{q}-$ and $10 \mathrm{q}$-specific sequences resided on chromosome 10 (Figure 3c).

A new muscle biopsy was performed and several unusual myopathological features were observed: central nucleation was marked, and peripherally placed sarcoplasmic masses and circular bundles of myofibrils existed (Figure 3d). Molecular genetic analysis revealed that the patient was heterozygous for the DMPK mutation; the copy number of CTG repeats in one chromosome was 90 (generally $<50$ copies in normal individuals; Figure $3 \mathrm{e}$ ). The patient was re-diagnosed with DM1, and the moderate expansion of CTG copies was consistent with her relatively mild clinical features.

\section{Family 4}

The proband $\left(\mathrm{II}_{2}\right)$, a 34-year-old woman, presented onset of symptoms at age 16, with a progressive inability to raise both arms above shoulder level, close her eyes and puff up her cheeks or smile. On first examination at age 29, she presented marked bifacial weakness and mild proximal muscle weakness with scapular winging in her upper limbs. From then on, the disease progressed very slowly for several years. Her CS score was 2.0 when she was 34 -years old. Her lower limb muscle bulk and strength were intact, and her gait was normal. Her CK was normal and the EMG showed myopathic changes in the proximal muscles of upper limbs. The left biceps muscle biopsy showed the presence of mildly degenerative and necrotic muscle fibers with secondary fibrosis, together with increased variation in myofiber size and the presence of inflammatory infiltrates of the vascular wall. She unequivocally fulfilled the clinical criteria of FSHD. ${ }^{2}$ Her mother $\left(\mathrm{I}_{2}\right)$ had a slowly progressing form of FSHD at age 20. Upon neurological examination at age 60, she had a CS score of 3.5. Her CK was slightly elevated (270 U/l). The proband's 36-year-old husband $\left(\mathrm{II}_{1}\right)$ and two daughters $\left(\mathrm{III}_{1}\right.$ and $\mathrm{III}_{2}, 10$ - and 8-years old, respectively) showed no clinical symptoms or signs of FSHD on physical and neurological examination. Their CK levels were normal.

Complementary allele typing and segregation analysis by PFGE revealed this pedigree with complex D4Z4 rearrangements (Figure 4a). The proband carried one 18 -kb $B \ln \mathrm{I}$-resistant D4Z4 repeat and three $B \ln \mathrm{I}$-sensitive D4Z4 repeat arrays of $47 \mathrm{~kb}, 83 \mathrm{~kb}$ and $102 \mathrm{~kb}$. The $18-\mathrm{kb}$ and $102-\mathrm{kb}$ arrays were inherited from her affected mother. Surprisingly, her husband carried four BlnI-resistant arrays of $24 \mathrm{~kb}$, $57 \mathrm{~kb}, 57 \mathrm{~kb}$ and $78 \mathrm{~kb}$, which indicated that two BlnI-resistant D4Z4 repeat arrays were mixtures of $4 \mathrm{q}-$ and $10 \mathrm{q}$-specific sequences resided on chromosome 10. Both daughters inherited the BlnI-resistant arrays of $24 \mathrm{~kb}$ from their father (Figure $4 \mathrm{~b}$ ). Based on these findings, the father and the two daughters could not be excluded as an asymptomatic FSHD case. Further, the 18 -kb array was identified as the $4 \mathrm{qA}$ variant, and the $24-\mathrm{kb}$ array was identified as the $4 \mathrm{qB}$ variant 


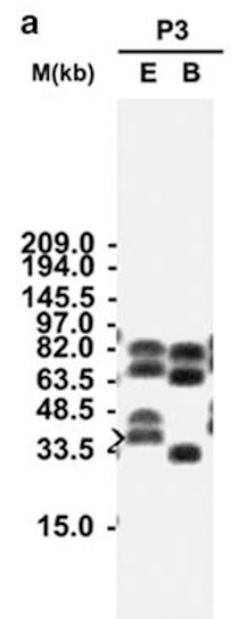

p13E-11

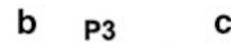

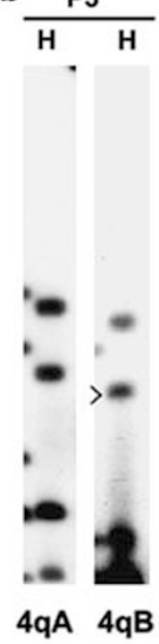

$\mathrm{c}$

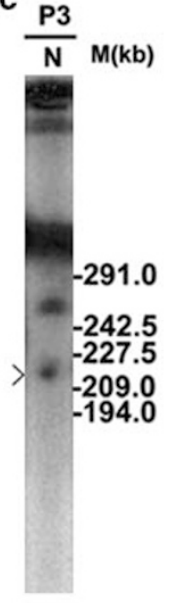

B31 d

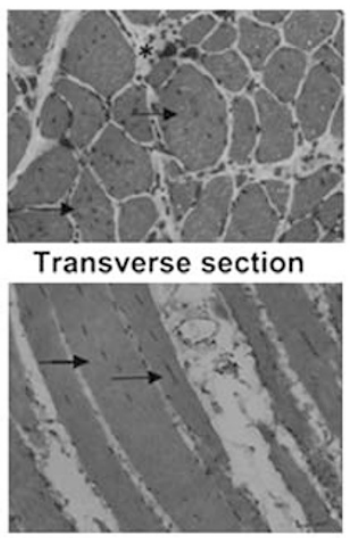

Longitudinal section e

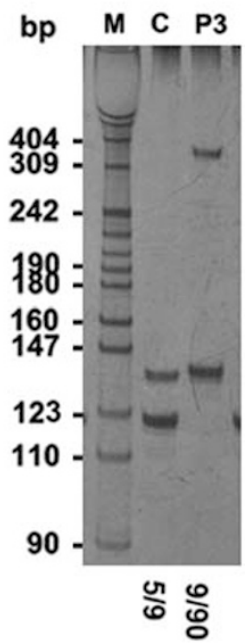

Figure 3 (a-c) PFGE-based Southern blot analysis of the proband (P3) from family 3. (a) P3 carried three BInl-resistant D4Z4 repeats of $36 \mathrm{~kb}$ (arrow), $76 \mathrm{~kb}, 86 \mathrm{~kb}$ and one BInl-sensitive repeat of $43 \mathrm{~kb}$. (b) Allelotyping analysis identified two $4 \mathrm{qA}$ variants of $43 \mathrm{~kb}$ and $86 \mathrm{~kb}$, and two $4 \mathrm{qB}$ variants of $36 \mathrm{~kb}$ (arrow) and $76 \mathrm{~kb}$. (c) Two Notl fragments of $218 \mathrm{~kb}(36 \mathrm{~kb}+182 \mathrm{~kb}$; arrow) and $258 \mathrm{~kb}(76 \mathrm{~kb}+182 \mathrm{~kb})$ demonstrated that $4 \mathrm{q}$-type alleles of $36 \mathrm{~kb}$ and $76 \mathrm{~kb}$ resided on chromosome 4 . So the extra $86 \mathrm{~kb}$ D4Z4 repeat was a mixture of 4q- and 10q-specific sequences resided on chromosome 10 . (d) Histopathological features of P3. Central nucleation is marked with an arrow ( $>50 \%)$. Peripherally placed sarcoplasmic masses and circular bundles of myofibrils were observed (asterisk). In addition, necrosis of single muscle fibers and many atrophic fibers was observed. HE, $\times 200$. (e) PAGE analysis of CTG repeats in the $3^{\prime}$ untranslated region of DMPK. The copy number of CTG repeats in one chromosome of P3 was 90 ((385-105)/3), indicating heterozygosity for the DMPK mutation. The exact numbers of CTG repeats are indicated below each lane. C, normal control; M, the size marker.

(Figure 4c). The chromosomal origin analysis revealed that the $18-\mathrm{kb}$ $4 \mathrm{qA}$ variant resided on chromosome 4 and the $24-\mathrm{kb} 4 \mathrm{qB}$ variant of $4 \mathrm{q} \rightarrow 10 \mathrm{q}$ sequence mixture resided on chromosome 10 . NotI fragments of $239 \mathrm{~kb}(57 \mathrm{~kb}+182 \mathrm{~kb})$ and $260 \mathrm{~kb}(78 \mathrm{~kb}+182 \mathrm{~kb})$ from $\mathrm{II}_{1}$ confirmed the presence of the $57-\mathrm{kb}$ and $78-\mathrm{kb} \mathrm{D} 4 \mathrm{Z} 4$ repeats on chromosome 4 , and another $57-\mathrm{kb}$ D4Z4 repeat was a $4 \mathrm{q} \rightarrow 10 \mathrm{q}$ sequence mixture (Figure $4 \mathrm{~d}$ ). Both daughters inherited a $47-\mathrm{kb}$ $10 \mathrm{q} \rightarrow 4 \mathrm{q}$ sequence mixture from their mother, indicating that they were normal.

\section{DISCUSSION}

Here, all three patients diagnosed with unrelated neuromuscular disorders had a $4 \mathrm{qB}$ variant smaller than $38 \mathrm{~kb}$ on chromosome 4 , which would have caused substantial diagnostic confusion in the absence of the $4 \mathrm{qA} / 4 \mathrm{qB}$ variant determination. Neuromuscular disorders that may be clinically and histopathologically similar to FSHD include the various limb-girdle and scapuloperoneal syndromes, which also may cause mild facial weakness. Ancillary investigations, such as the assessments of CK, EMG and muscle biopsy, are often insufficient for diagnosis, as was the case in the probands from families 1 and 2. However, subsequent DNA analysis of the nonpathogenic short $4 \mathrm{qB}$ variant excluded the diagnosis of FSHD, and western blot analysis further confirmed the diagnosis of LGMD2 in these patients. The proband from family 3 also presented some FSHDlike symptoms and signs based on clinical observation, such as prominent involvements of the facial muscles and mild shoulder girdle and pelvic girdle weakness. The facial-sparing scapular myopathy was described as the most common clinical variant of FSHD, and some atypical phenotypes including LGMD, distal myopathy, mild or partial forms have been described in several papers published before 2004. ${ }^{18-20}$ However, the identification of the $4 \mathrm{qA}$ and $4 \mathrm{qB}$ variants had not been performed in these special cases. Here, the D4Z4 contraction in the proband from family 3 was confirmed as linked to the $4 \mathrm{qB}$ variant and considered as nonpathogenic.
We had previously demonstrated the presence of an FSHD-sized $4 \mathrm{qB}$ variant on chromosome 4 in healthy individuals. ${ }^{21}$ In the present study, we provided further evidence of the nonpathogenic $4 \mathrm{qB}$ variant in other neuromuscular disorders. This may also explain the rare finding of FSHD-sized D4Z4 repeats that do not co-segregate with unrelated disorders. ${ }^{22}$ Therefore, the extension of the clinical spectrum of FSHD needs to be carefully evaluated. Although some rare cases with 'double trouble' had been reported, ${ }^{11,23}$ NotI digestions followed by hybridizations with B31 need to conclusively identify the correct chromosome 4, and some complex genetic constitutions with exchanged D4Z4 alleles of chromosome 4 and chromosome 10 origins are presented below.

There are two uncommon factors that made the genetic confirmation of the fourth pedigree confusing. First, both 4qA- and 4qB-type FSHD-sized D4Z4 alleles were found in the same family. Second, complex genetic constitutions were observed in the same family. Before complementary allelotyping analysis and integrated chromosome assignments, the members of the family who carried 4qB-type FSHD-sized alleles had been misdiagnosed as asymptomatic cases. Through PFGE-based DNA analysis with probes $4 \mathrm{qA}, 4 \mathrm{qB}$ and $\mathrm{B} 31$, we confirmed that the $18-\mathrm{kb} 4 \mathrm{qA}$ variant correlated with FSHD in the proband and that the one carried by her mother resided on chromosome 4 , whereas the $24-\mathrm{kb} 4 \mathrm{qB}$ variant, which is considered as nonpathogenic, in the healthy husband and the two daughters resided on chromosome 10. Moreover, we were able to confirm the complex D4Z4 rearrangements of two exchanged repeat arrays, one on chromosome 4 and one on chromosome 10, inherited from the maternal $10 q \rightarrow 4 q$ repeat and from the paternal $4 q \rightarrow 10 q$ repeat. Although the haplotypes composed of a mixture of $4 \mathrm{q}$ and $10 \mathrm{q}$ sequences were detected at frequencies $>15 \%$ in the Chinese FSHD, ${ }^{12}$ such exchanged constitution cannot be identified by conventional DNA diagnosis and never been observed in the Chinese population. As reported, all 4q subtelomeres originate from only four discrete interchromosomal sequence transfers during human evolu- 
a

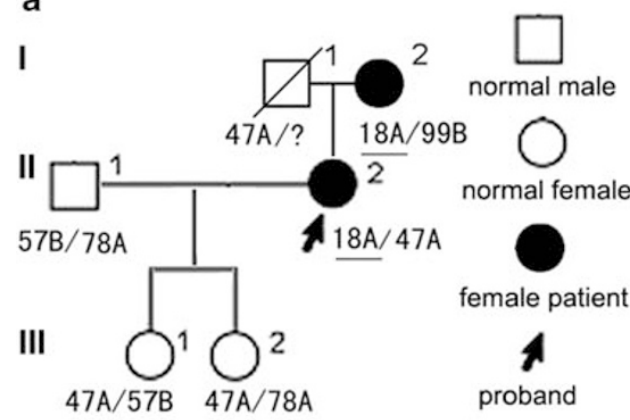

II1

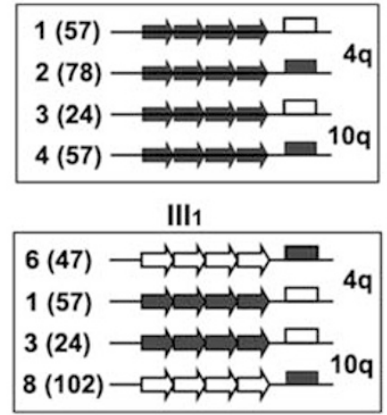

II2

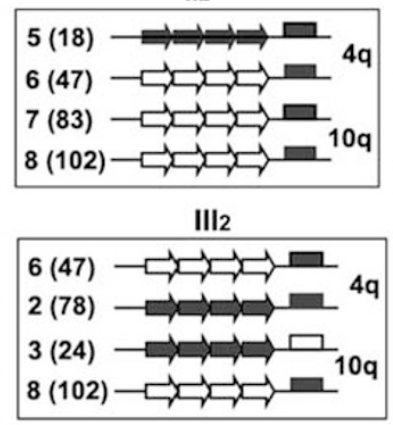

BInl-resistant repeat

$\Rightarrow \overrightarrow{x \rightarrow y} \Rightarrow$

BInl-sensitive repeat

且

4qA variant

口․

$4 \mathrm{qB}$ variant

(...)

repeat length (kb) b

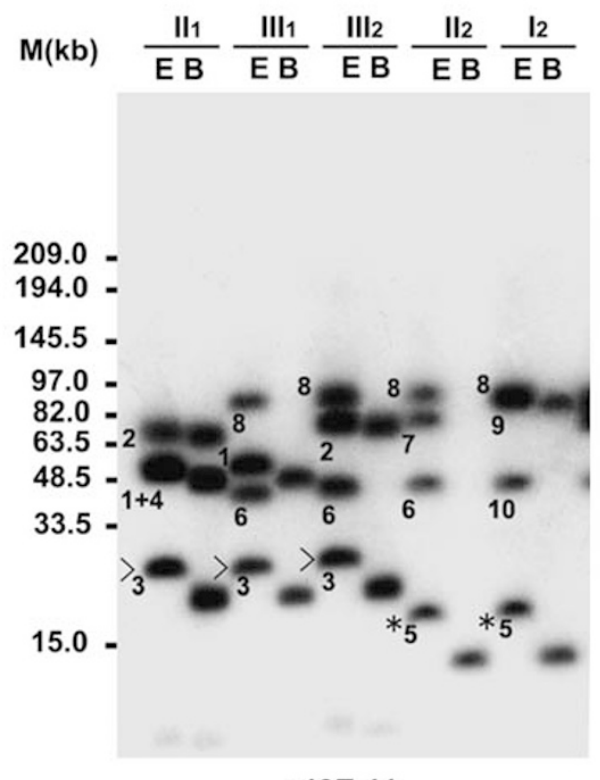

c

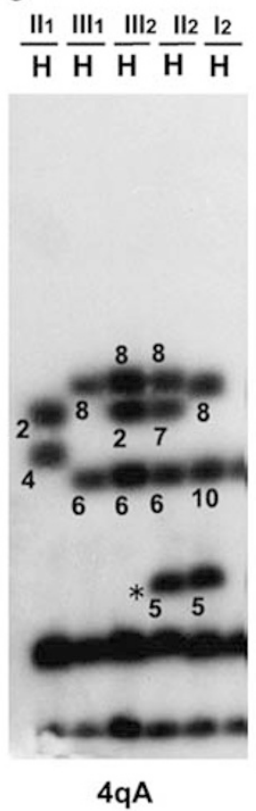

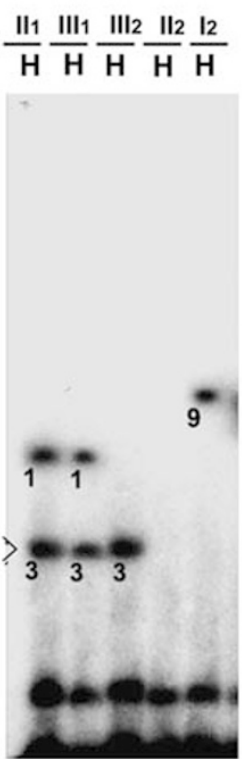

$4 q B$ d

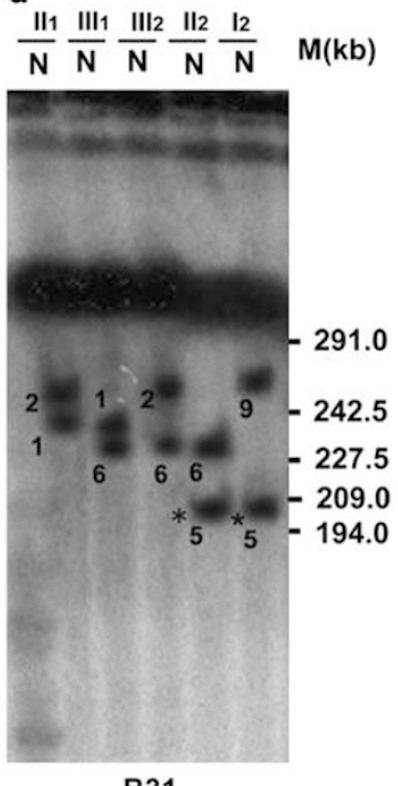

Figure 4 PFGE-based Southern blot analysis of the family 4 with complex genetic constitutions. (a) Schematic diagram showing the genetic arrangement of $4 \mathrm{q}$ and $10 \mathrm{q}$ alleles in this pedigree. (b) The proband $\left(\mathrm{II}_{2}\right)$ carried one BInl-resistant D4Z4 repeat of $18 \mathrm{~kb}(5)$ and three BInl-sensitive D4Z4 repeat arrays of $47 \mathrm{~kb}(6), 83 \mathrm{~kb}(7)$ and $102 \mathrm{~kb}(8)$, indicating one $10 \mathrm{q} \rightarrow 4 \mathrm{q}$ sequence mixture resided on chromosome 4 . The 18-kb fragment and 102-kb fragment were inherited from the patient's affected mother $\left(\mathrm{I}_{2}\right)$ who carried another BInl-resistant D4Z4 repeat of $99 \mathrm{~kb}(9)$ and BInl-sensitive D4Z4 repeat of $49 \mathrm{~kb}(10)$. The husband $\left(\mathrm{II}_{1}\right)$ carried four BInl-resistant D4Z4 repeat arrays of $24 \mathrm{~kb}(3), 57 \mathrm{~kb}, 57 \mathrm{~kb}(1+4$, comigrating alleles) and $78 \mathrm{~kb}(2)$, indicating that two $B / \mathrm{nl}$ resistant D4Z4 repeat arrays were mixtures of $4 \mathrm{q}$ - and $10 \mathrm{q}$-specific sequences resided on chromosome 10 . Two daughters $\left(\mathrm{III} \mathrm{I}_{1}\right.$ and $\left.\mathrm{III}_{2}\right)$ inherited the FSHDsized allele of $24 \mathrm{~kb}$ from their father. (c) The 18-kb fragment was identified as the $4 \mathrm{qA}$ variant, whereas the 24-kb fragment was identified as the $4 \mathrm{qB}$ variant. (d) The 18-kb $4 q A$ variant resided on chromosome 4 , whereas the $24-\mathrm{kb} 4 \mathrm{qB}$ variant was a likely $4 \mathrm{q} \rightarrow 10 \mathrm{q}$ sequence mixture resided on chromosome 10 owing to its disappearance on Notl-digestion. Two Notl fragments of $239 \mathrm{~kb}(57 \mathrm{~kb}+182 \mathrm{~kb})$ and $260 \mathrm{~kb}(78 \mathrm{~kb}+182 \mathrm{~kb})$ were detected in the husband, confirming that the 57-kb and 78-kb fragments, respectively, resided on chromosome 4 and were inherited by his daughters. Thus, another $B / n \mid$-resistant D4Z4 repeat of $57 \mathrm{~kb}$ resided on chromosome 10 . Both daughters inherited the $47 \mathrm{~kb}$ fragment $(10 \mathrm{q} \rightarrow 4 \mathrm{q})$ from their mother. The $83 \mathrm{~kb}$ $B / n \mid$-sensitive D4Z4 repeat resided on chromosome 10 of the proband was of paternal origin. The FSHD-causing 4qA variant (asterisk) is underlined, and the FSHD-sized $4 \mathrm{qB}$ variant is indicated by an arrow.

tion, and haplotypes with mixtures of $4 \mathrm{q}-$ and $10 \mathrm{q}-$-specific sequences represent intermediate structures in the transition from $4 \mathrm{q}$ to $10 \mathrm{q}$ subtelomeres. ${ }^{10}$ We also found that in this family, a $4 \mathrm{qB}$ variant resided on chromosome 10, confirming the same detection in other populations, ${ }^{10}$ and further suggesting that the breakpoint of the D4Z4 repeat was distal to the $4 \mathrm{qA}$ and $4 \mathrm{qB}$ variants. The pathophysiological pathway from $4 \mathrm{q} 35$ deletion together with the $4 \mathrm{qA}$ variant to the clinical picture of FSHD is unclear.

In summary, this is the first study to confirm that D4Z4 contractions on the $4 \mathrm{qB}$ variant do not cause FSHD in the Chinese population. Furthermore, our results emphasize that the D4Z4 repeat length analysis alone is insufficient for the diagnosis of FSHD, especially when used as an exclusion criterion. This analysis should be accompanied by $4 \mathrm{qA} / 4 \mathrm{qB}$ variant determination and integrated chromosome assignments, especially in patients with obscure and unclassified myopathies similar to atypical forms of FSHD.

\section{CONFLICT OF INTEREST}

The authors declare no conflict of interest.

\section{ACKNOWLEDGEMENTS}

We sincerely thank the participants for their help and willingness to participate in this study and the anonymous reviewers for helping to improve this paper. This work was supported by Grant 30570652 from the National Natural 
Science Foundation (Beijing), a grant from the Huashan Hospital for the special professorship of Fudan University (Shanghai), the key program 09ZD005 of scientific research of Fujian Medical University (Fuzhou), Grant 2006J0007 from the Natural Science Foundation of Fujian Province, China. SvdM is supported by the Fields Center for FSHD and Neuromuscular Research (http://www.urmc.rochester.edu/fields-center/) and by the Netherlands Organization for Scientific Research NWO (016.056.338). Study concept and design (Dr Wu); acquisition of data (Drs Z-Q Wang, $\mathrm{N}$ Wang, Murong and $\mathrm{Wu}$ ); analysis and interpretation of data (Drs Z-Q Wang, $\mathrm{N}$ Wang, van der Maarel and Wu); drafting of the paper (Drs Z-Q Wang and $\mathrm{Wu}$ ); critical revision of the paper for important intellectual content (Drs van der Maarel and Wu); obtained funding (Drs N Wang and Wu); administrative, technical or material support (Drs Z-Q Wang, N Wang, van der Maarel and $\mathrm{Wu})$; study supervision (Dr Wu).

1 Padberg GW: Facioscapulohumeral Disease: MD Thesis. Leiden, The Netherlands: Leiden University, 1982.

2 Padgerg GW, Lunt PW, Koch M et al: Facioscapulohumeral muscular dystrophy. In: Emery AEH (ed).: Diagnostic Criteria for Neuromuscular Disorders, 2nd edn. London England: Royal Society of Medicine Press, 1997; 9-16.

3 Wijmenga C, Hewitt JE, Sandkuijl LA et al: Chromosome 4q DNA rearrangements associated with facioscapulohumeral muscular dystrophy. Nat Genet 1992; 2: 26-30.

4 van Deutekom JCT, Wijmenga C, Van Tienhoven EAE et al: FSHD associated DNA rearragements are due to deletions of intergral copies of a $3.2 \mathrm{~kb}$ tandemly repeated units. Hum Mol Genet 1993; 2: 2037-2042.

5 Lunt PW: 44th ENMC International Workshop: Facioscapulohumeral Muscular Dystrophy: Molecular Studies 19-21 July 1996, Naarden, The Netherlands. Neuromuscu Disord 1998; 8: 126-130.

6 Lemmers RJ, de Kievit P, Sandkuijl L et al: Facioscapulohumeral muscular dystrophy is uniquely associated with one of the two variants of the $4 \mathrm{q}$ subtelomere. Nat genet 2002; 32: 235-236.

7 Lemmers RJ, Wohlgemuth M, Frants RR, Padberg GW, Morava E, van der Maarel SM: Contractions of D4Z4 on $4 \mathrm{qB}$ subtelomeres do not cause facioscapulohumeral muscular dystrophy. Am J Hum Genet 2004; 75: 1124-1130.

8 Thomas NS, Wiseman K, Spurlock G, MacDonald M, Ustek D, Upadhyaya M: A large patient study confirming that facioscapulohumeral muscular dystrophy (FSHD) disease expression is almost exclusively associated with an FSHD locus located on a 4qA-defined 4qter subtelomere. J Med Genet 2007; 44: 215-218.

9 Lemmers RJ, Wohlgemuth M, van der Gaag KJ et al: Specific sequence variations within the $4 \mathrm{q} 35$ region are associated with facioscapulohumeral muscular dystrophy. Am J Hum Genet 2007; 81: 884-894.

10 Lemmers RJ, van der Vliet PJ, van der Gaag KJ et al: Worldwide population analysis of the $4 \mathrm{q}$ and $10 \mathrm{q}$ subtelomeres identifies only four discrete interchromosomal sequence transfers in human evolution. Am J Hum Genet 2010; 86: 364-377.

11 Sacconi S, Salviati L, Bourget I et al: Diagnostic challenges in facioscapulohumeral muscular dystrophy. Neurology 2006; 67: 1464-1466.

12 Wu ZY, Wang ZQ, Murong SX, Wang N: FSHD in Chinese population: characteristics of translocation and genotype-phenotype correlation. Neurology 2004; 63: 581-583.

13 Wang ZQ, Wu ZY, Wang N et al: Characteristics of gene structure in FSHD-related 4q35 subtelomere and genotype-phenotype correlation in Chinese Han Population. Zhonghua Yi Xue Za Zhi 2009; 89: 304-310. (in Chinese).

14 Ricci E, Galluzzi G, Deidda G et al: Progress in the molecular diagnosis of facioscapulohumeral muscular dystrophy and correlation of the number of $\mathrm{Kpnl}$ repeats at the 4q35 locus and clinical phenotype. ANN Neurol 1999; 45: 751-757.

15 Lemmers RJ, Osborn M, Haaf T et al: D4F104S1 deletion in facioscapulohumeral muscular dystrophy: phenotype, size, and detection. Neurology 2003; 61: 178-183.

16 Hack AA, Lam MY, Cordier $L$ et al: Differential requirement for individual sarcoglycans and dystrophin in the assembly and function of the dystrophin-glycoprotein complex. J Cell Sci 2000; 113: 2535-2544.

17 Comerlato EA, Scola RH, Werneck LC: Limb-girdle muscular dystrophy: an immunohistochemical diagnostic approach. Arq Neuropsiquiatr 2005; 63: 235-245.

18 Felice KJ, North WA, Moore SA, Mathews KD: FSH dystrophy 4q35 deletion in patients presents presenting with facial-sparing scapular myopathy. Neurology 2000; 54: 1927-1931.

19 Krasnianski M, Eger K, Neudecker S, Jakubiczka S, Zierz S: Atypical phenotypes in patients with facioscapulohumeral muscular dystrophy 4q35 deletion. Arch Neurol 2003; 60: 1421-1425.

20 van der Kooi AJ, Visser MC, Rosenberg $\mathrm{N}$ et al: Extension of the clinical range of facioscapulohumeral dystrophy: report of six cases. J Neurol Neurosurg Psychiatry 2000; 69: 114-116.

21 Chen ZJ, Wang ZQ, Wu ZY, Wang N, Lin MT, Murong SX: Analysis of alleles 4qA and $4 \mathrm{qB}$ of the chromosome $4 \mathrm{q}$ subtelomere in Chinese Han population. Zhonghua $Y i X_{u}$ Yi Chuan Xue Za Zhi 2007; 24: 334-337. (in Chinese).

22 Auer-Grumbach M, Wagner K, Strasser-Fuchs S et al: Clinical predominance of proximal upper limb weakness in CMT1A syndrome. Muscle Nerve 2000; 23: 1243-1249.

23 Felice KJ, Johns JM, Conway SR: Facioscapulohumeral dystrophy presenting as infantile facial diplegia and limb-girdle myopathy in members of the same family. Muscle Nerve 2005; 32: 368-372. 